\title{
STUDY OF SECONDARY EMISSION ENHANCED PHOTOINJECTOR*
}

\author{
Xiangyun Chang, Ilan Ben-Zvi, Andrew Burrill, Peter Johnson, Jorg Kewisch, Triveni Rao, \\ Zvi Segalov, Yongxiang Zhao, BNL, Upton, NY 11973 U.S.A.
}

\section{Abstract}

The secondary emission enhanced photoinjector (SEEP) is a very promising new approach to the generation of high-current, high-brightness electron beams. Primary electrons with a few thousand electronvolts of energy strike a specially prepared diamond window. The large Secondary Electron Yield (SEY) provides a multiplication of the number of electrons by about two orders of magnitude. The secondary electrons drift through the diamond under an electric field and emerge into the accelerating proper of the "gun" through a Negative Electron Affinity (NEA) surface of the diamond (Hydrogen terminated). We present the calculation of heating power sources and the temperature distribution in detail. Some properties of the secondary electron beam related to beam dynamics are also reported. The results demonstrate the feasibility of this kind of cathode.

\section{INTRODUCTION}

The creation of high average-current, high brightness electron beams is a key technology for a large number of accelerator-based systems, such as ultra-high-power FreeElectron Lasers (FELs), Energy-Recovery Linac (ERL) light sources, electron cooling of hadron accelerators, and many more.

Fig.1 shows a schematic layout of such a device.

The laser light illuminates on a high-QE photocathode, such as $\mathrm{CsK}_{2} \mathrm{Sb}$. The primary electrons produced on the photocathode are accelerated to a few thousand electron- volts by the DC field applied between the photocathode and a diamond window. The primary electrons penetrate the thin metal film deposited on the backside (left side) of the diamond window and produce a large number of secondary electrons in the diamond. The Secondary Electron Yield (SEY) can be more than 100. The secondary electrons drift through the diamond under a high RF electric field and emerge into the accelerating proper of the "gun" through a Negative Electron Affinity (NEA) [1] surface of the diamond.

The heat dissipation is an important problem in the application of the diamond cathode. We present the calculation of heating power sources and the temperature distribution in detail. Some properties of the secondary electron beam related to beam dynamics are also reported. The results demonstrate the feasibility of this kind of cathode.

Naturally, this cathode will be most suitable for a superconducting RF gun for $\mathrm{CW}$ operation, but it has significant advantages also for normal conducting, pulsed RF guns and DC guns. Therefore, in the following, we will describe the application of this new paradigm as applied to a superconducting RF gun working at $703 \mathrm{MHz}$ which is the RF frequency of electron cooling project for Relativistic Heavy Ion Collider (RHIC). Extensions to the others are trivial.

\section{DESIGN CONSIDERATIONS}

Diamond has very wide bandgap $(5.47 \mathrm{~V})$ between conduction band and valence band at $300 \mathrm{~K}$. This makes

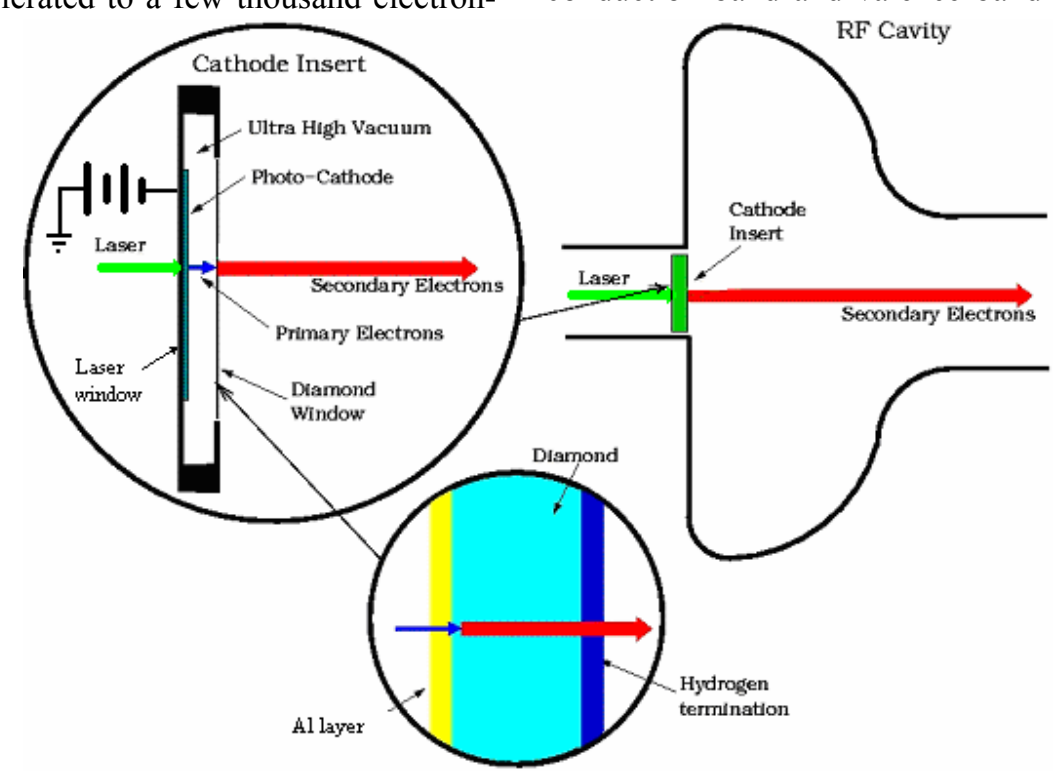

Figure 1: The schematic of the secondary emission enhanced photo-injector

* Work performed under the auspices of the U.S. Department of Energy and partially funded by the US Department of Defense diamond a good insulator with very high breakdown field $(1000 \mathrm{MV} / \mathrm{m})$ and a material easy to have NEA. 
The metal layer is used to replenish the secondary electrons and provide the RF current. One would like to use a material, which has an electrical conductivity $(\sigma)$ as high as possible to reduce the resistance of the film. Also one would like the material to have a Continuous Slowing Down Approximation (CSDA) range parameter $\left(\mathrm{R}_{\mathrm{CSDA}}\right)$ as long as possible to reduce the energy loss in the metal film. The choice of the material is determined by the parameter $\sigma \times R_{C S D A}$ (the higher the better). Possible material choices are aluminum (Al), Copper, Silver and Gold. Aluminium has the highest $\sigma \times R_{C S D A}$. We will assume aluminum to be the metal layer material on the diamond film.

Assume the primary electron energy is $10 \mathrm{keV}$, the aluminum thickness $\left(t_{\mathrm{Al}}\right)$ can be as high as $800 \mathrm{~nm}$ and the average primary electron energy after the film is still of the order of $4 \mathrm{keV}$, which is in the acceptable range of the primary electron energy on diamond. Therefore the aluminum thickness is chosen to be $800 \mathrm{~nm}$. Although the aluminum thickness is much less than the $703 \mathrm{MHz}$ RF skin depth $(3.1 \mu \mathrm{m})$, the RF penetration of the aluminum film is very poor [2]. In other words, the strong RF current will all go through the Al film. This makes a thicker Al film very important.

SEY is another important parameter designing the system. Higher SEY will need less primary electron current and power. It is reported that the average energy loss of a primary electron for the generation of one electron-hole pair is about $13 \mathrm{eV}$. The SEY coefficient would be about 300 under above conditions.

The diamond window plays a role not only in the production of the secondary electrons but also in the heat dissipation. Diamond has the highest thermal conductivity, which makes it possible to handle high power with a thin film. One would like to use a thicker diamond to improve the thermal conduction of the window. But for RF application the diamond thickness is limited by the allowed transmission time for the secondary electrons and the electron drifting velocity in diamond.

The electron drift velocity in diamond under high external electric field has been experimentally measured. The data shows that when the external field is larger than $2 \mathrm{MV} / \mathrm{m}$ the electron drift velocity is saturated at $2.7 \times 10^{5} \mathrm{~m} / \mathrm{s}$ and is independent of temperature. This means that the secondary electron bunch drift velocity in diamond is almost constant except when the charge density is very high and the space charge force is comparable to the RF field. Assume the allowed transmission time through diamond is $30 \mathrm{deg}$., the diamond thickness for our $703 \mathrm{MHz}$ RF gun is estimated to be about $32 \mu \mathrm{m}$.

To estimate the diamond temperature one needs also to know the diamond thermal conductivity. The diamond thermal conductivity as a function of Temperature can be expressed by the Debye model [3]. It is determined by the crystal size, Umklapp process, ${ }^{13} \mathrm{C}$ isotopes scattering and
Impurity scattering. The crystal size is the most decisive parameter. We will conservatively adopt $10 \mu \mathrm{m}$ as the grain size in our example. The maximum thermal conductivity is about $1300 \mathrm{~W} / \mathrm{mK}$ at $200 \mathrm{~K}$.

The most important impurities in diamond are Boron (p-type) and Nitrogen (n-type). There are three main effects of the impurities; heating of diamond, trapping of charge carriers and field shielding. A nitrogen impurity has activation energy higher than $1.7 \mathrm{eV}$ and is hard to be ionized at room temperature. A boron impurity has an activation energy ranges from $\sim 0.1 \mathrm{eV}$ to $\sim 0.37 \mathrm{eV}$ depending on the doping concentration and as such is very easily ionized. The Boron concentration needs to be much less than $1 \mathrm{ppm}$ to reduce the heating of diamond. The Nitrogen impurity also forms hole traps while the Boron impurity forms electron traps. The grain boundaries concentrate charge carrier traps. The trap centres trap the charge carrier and therefore generate space charge and reduce the external field. It is therefore desirable to use the high purity single crystal diamond.

\section{DIAMOND TEMPERATURE}

The heating of the diamond cathode comes from:

- The primary electron power.

- The power that secondary electron transmitting diamond under strong RF field.

- Electron replenishment power generated on aluminum film.

- RF power on aluminum film.

- The movement of the impurity induced free electrons in the diamond conduction band (Nitrogen doping) and holes in the valence band (Boron doping) in the strong RF field.

There is also the power from electron replenishment in the diamond. As the replenishment distance is very small $(\sim 1 \mu \mathrm{m})$, this effect is ignored.

A FORTRAN code is written to simulate the temperature distribution in diamond including all the factors discussed above. The criterion is that the maximum temperature in diamond cathode be well below $1000^{\circ} \mathrm{C}$ to avoid significant Hydrogen desorbtion from the surface [4].

The following is an example of the SEEP application on the RHIC electron cooling project [5]. It requires a large charge per bunch $(\sim 20 \mathrm{nC} /$ bunch $)$, but a relatively low repetition frequency $(9.4 \mathrm{MHz})$, i.e., low average current. This requires a large cathode radius $(\mathrm{R} \sim 10 \mathrm{~mm})$ to reduce space charge force.

Fig. 2 is the temperature distribution from the center of the diamond $(\mathrm{R}=0)$ to the diamond edge. We assume that the primary electron energy is $10 \mathrm{keV}$, the thickness of the diamond window is $30 \mu \mathrm{m}$, the thickness of the aluminum layer is $800 \mathrm{~nm}$, the peak RF field on cathode $\mathrm{E}_{0}=15 \mathrm{MV} / \mathrm{m}, \mathrm{SEY}=300$. The temperature at the diamond edge is fixed to $80 \mathrm{~K}$ (assuming Liquid Nitrogen (LN) cooling) and the primary electron pulse length is $10 \mathrm{deg}$. The dependence of the temperature on the radius is shown for different cathode radii. 


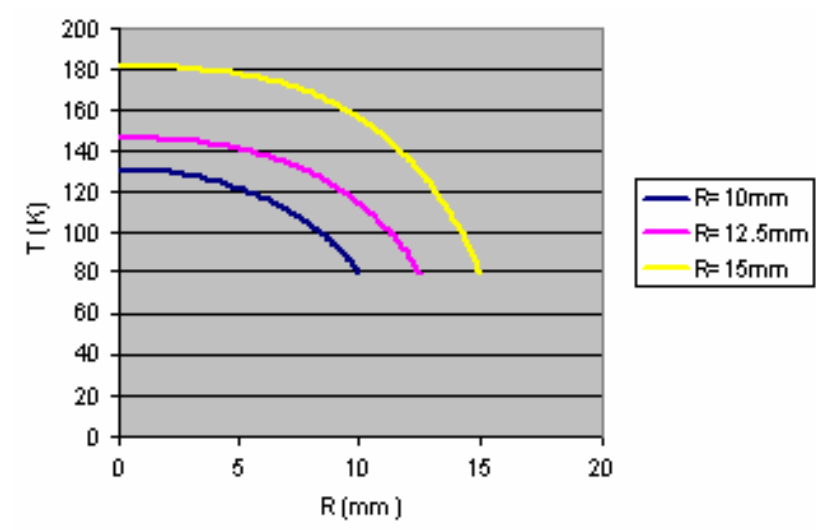

Figure 2: Temperature vs. R for RHIC electron cooling proiect

Table 1: Power from different sources

\begin{tabular}{|l|l|l|l|}
\hline R & $\mathbf{1 0 m m}$ & $\mathbf{1 2 . 5} \mathbf{m m}$ & $\mathbf{1 5 m m}$ \\
\hline Primary & $6.3(\mathrm{~W})$ & $6.3(\mathrm{~W})$ & $6.3(\mathrm{~W})$ \\
\hline Secondary & $7.6(\mathrm{~W})$ & $7.6(\mathrm{~W})$ & $7.6(\mathrm{~W})$ \\
\hline RF & $7.5(\mathrm{~W})$ & $20.0(\mathrm{~W})$ & $48.6(\mathrm{~W})$ \\
\hline Replenishment & $0.042(\mathrm{~W})$ & $0.046(\mathrm{~W})$ & $0.054(\mathrm{~W})$ \\
\hline Total & $21.4(\mathrm{~W})$ & $33.9(\mathrm{~W})$ & $62.5(\mathrm{~W})$ \\
\hline
\end{tabular}

The powers from different sources are listed in table 1 .

We see that the heat power is dominated by RF heat when $\mathrm{R}>12 \mathrm{~mm}$. However, with a radius of $15 \mathrm{~mm}$ the temperature of the cathode is still below $300 \mathrm{~K}$

\section{SECONDARY ELECTRON BEAM QUALITY}

The secondary bunch lengthening while drifting in diamond is small because of the saturation of the drifting velocity in a strong field. The bunch lengthening mainly comes from $\mathrm{R}_{\mathrm{CSDA}}$ straggling. If one assumes the probability distribution of the ranges about the mean $\mathrm{R}_{\mathrm{CSDA}}$ is a Gaussian distribution, the straggling distance of primary electrons in our example is calculated to be about 3ps.

Another important parameter is the secondary electron temperature. This can be obtained by solving 2 equations. One is the equation for the equilibrium electron random energy as a function of the inelastic mean free path (IMFP) $\lambda_{i}$, the lattice temperature $T_{L}$ and the electric field in the diamond $\mathrm{E}_{0}$ :

$$
-\mathrm{eE}_{0} \mathrm{v}_{\mathrm{D}}-\frac{\overline{\mathrm{W}}\left(\mathrm{T}_{\mathrm{e}}\right)-\overline{\mathrm{W}}\left(\mathrm{T}_{\mathrm{L}}\right)}{\tau_{\mathrm{W}}}=0
$$

where $W\left(T_{e}\right)$ and $W\left(T_{L}\right)$ are electron thermal energy and lattice thermal energy, $\tau_{\mathrm{W}}=\lambda_{\mathrm{i}} / \mathrm{v}_{\mathrm{e}}$ is the energy relaxation time and $\mathrm{v}_{\mathrm{e}}=\sqrt{2 \overline{\mathrm{W}}\left(\mathrm{T}_{\mathrm{e}}\right) / \mathrm{m}_{\mathrm{e}}}$ is the electron thermal velocity.

Another is the IMFP semi-empirical formula [6]:

$$
\lambda_{i}=\left[538 E_{r}^{-2}+0.41\left(a_{m} E_{r}\right)^{\frac{1}{2}}\right] a_{m}
$$

where $a_{m}$ is the thickness of a monolayer in nanometers, for diamond $\mathrm{a}_{\mathrm{m}}=0.1783 \mathrm{~nm}$. $\mathrm{E}_{\mathrm{r}}$ is the electron's energy above the Fermi level.

The electron thermal energy under $10 \mathrm{MV} / \mathrm{m}$ is found out to be about $0.4 \mathrm{eV}$.

\section{CONCLUSIONS}

The mechanics, physical properties and the electron beam qualities of the secondary emission enhanced photoinjector are presented. The results show great feasibility of this kind of cathode. The cathode can easily handle $100 \mathrm{~W}$ of heat power with its maximum temperature well below the criterion. The secondary electron beam quality is comparable to the traditional photocathode.

\section{REFERENCES}

[1] J. E. Yater, A. Shih, and R. Abrams, "Electron transport and emission properties of C(100)", Physical Review B, V56, R4410.

[2] Yongxiang Zhao, Ilan Ben-Zvi, Xiangyun Chang, Triveni Rao, Wei Chen, Robert Di Nardo and Rolf Beuttenmuller, "The Penetrability of a thin Metallic Film Inside the RF Field", PAC'05, Knoxville, Tennessee, U.S.A. May 2005, http://www.jacow.org.

[3] E. Worner*, E. Pleuler, C. Wild, P. Koidl, "Thermal and optical properties of high purity CVD-diamond discs doped with boron and nitrogen", Diamond and Related Materials 12 (2003) 744-748.

[4] Brian D. Thorns, Pehr E. Pehrsson, and James E. Butler, "A vibrational study of the adsorption and desorption of hydrogen on polycrystalllne diamond", J. Appl. Phys. 75 (3) 1804, 1 February 1994.

[5] V. Parkhomchuk, I. Ben-Zvi, "Electron Cooling for RHIC", C-A/AP/47, April 2001.

[6] M. P. Seah and W. A. Dench, Surf. Interface Anal. 1, pg10 (1979). 